Proverecioness

Sis. 16. X" l. pp. 23-36. . July 1997

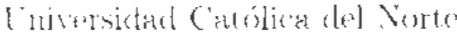

DOI: 10.22199/S07160917.1997.0001.00003

Antofingrinta - Chilf

\title{
ON EUCLIDEAN RINGS
}

\author{
Ahmet Goksel Agargun \\ Yildiz Technical University. Sisli-Istambul. Turkey
}

\begin{abstract}
In this urficle we ertend the concept of Euclidean rings and Motzhin's wort to commutatue rings (not necessorily with an identity).
\end{abstract}




\section{Introduction}

In 1949 Motzkin [8] had proved a necessary and sufficient condition for an integral domain to be a Eucliclean ring. Two generalizations of this concept (anle in 1969 and 1971. Firstly. Fletcher gave the Euclidean property for a commutative ring with identity in which zero divisors nay appear [3]. Thum sammel postulated whether a mapping into a general well-ordered sot would increase the number of Euclidean rings [12]. In 1977, the first example of a Eucliclean domain without any integer-valued algorithm was given by Hiblot [4]. [5]. In 1978. Nagata [9] also proved that there exists an integral domain which is a Euclidean ring with a mapping into a wellordered set but not a Euclidean ring with a mapping into $\mathrm{Z}^{+} \cup\{0\}$. In 1985 Ninata ${ }^{\prime} 10^{\prime}$. gave his definition which leses an algorithm which maps into a partially ordered set with the minimum condition. But this definition does not inc'reast' the fannily of Euclidean rings given by Samuel's definition.

We generalize the concept of Euclidean rings to commutative rings which do not neccssarily have an identity. We give seven new definitions and the relations among then. Our definitions are equivalent to the usual definit ions in the ("ase of a ring with identity. We generalize Motzkin's work 11) give a useful result for finding the Euclidean algorithm (smallest algorithun) for (ommutative rings. Also some examples of Euclidean rings and the smallest algorithms are given in this work.

\section{The Euclidean Algorithms}

In generalising the concept of a Euclidcan ring to commutative rings we take the opportmity of examining the connections between the many different detinitions. Let $R$ and $R^{\prime}$ be commutative rings, $R^{\prime}$ with identity and let $\theta: \mathrm{R} \rightarrow \mathrm{R}^{\prime}$ be a monomorphisn where $\theta(\mathrm{R})$ is an ideal of $\mathrm{R}^{\prime}$. Let us call this kind of mononorphism as a $G$-monomorphism. Suppose $a, b \in R, W$ is a woll-ordered set and $\varphi$ is mapping into $W$. The three areas for choice are

(i) $\uparrow: \mathrm{R} \backslash\{0\} \rightarrow \mathrm{W}$ or $\varphi: \mathrm{R} \rightarrow \mathrm{W}$.

(ii) b) any alement of $\mathrm{R}$ or $\mathrm{b} \neq 0$,

(iii) $\theta(\mathrm{a})=\mathrm{q}^{\prime} \theta(\mathrm{b})+\theta(\mathrm{r})$ where $\mathrm{r}=0$ or $\varphi(\mathrm{r})<\varphi(\mathrm{b})$, or

$\mathrm{r}=\mathrm{b}$ or $\varphi(r)<\varphi(\mathrm{b})$, or

$\varphi(r)<\varphi(b)$.

Therefore we have twelve possible definitions, but some of these do not give an algorithm. 
Definition 2.1 A map $\psi: \mathrm{R} \backslash\{0\} \rightarrow \mathrm{W}$ is called a Euclidean algorithm (with respect to $\theta: R \rightarrow R^{\prime}$ ) if for all $a, b \in R, b \neq 0$, there exist $q \in R$ and $r \in R$ such that

$$
\theta(\mathrm{a})=\mathrm{q}^{\prime} \theta(\mathrm{b})+\theta(\mathrm{r}) \text { where } \mathrm{r}=0 \text { or } \varphi(\mathrm{r})<\varphi(\mathrm{b}) .
$$

Sinilarly we have the other definitions by consideration the three areas for chosice.

Definition $22 \quad \varphi: \mathrm{R} \backslash\{0\} \rightarrow \mathrm{W}, \mathrm{b} \neq 0, \mathrm{r}=\mathrm{b}$, or $\varphi(r)<\varphi(\mathrm{b})$.

Definition $23 \quad \varphi: \mathrm{R} \rightarrow \mathrm{W}, \mathrm{b} \neq 0, \mathrm{r}=0$, or $\varphi(r)<\varphi(\mathrm{b})$.

Definition $2.4 \varphi: \mathrm{R} \rightarrow \mathrm{W}, \mathrm{b} \neq 0, \mathrm{r}=\mathrm{b}$, or $\varphi(r)<\varphi(\mathrm{b})$.

Definition $2.5 \quad \varphi: \mathrm{R} \rightarrow \mathrm{W}, \mathrm{b} \neq 0, \varphi(r)<\varphi(\mathrm{b})$.

Definition $2.6 \quad \omega: \mathrm{R} \rightarrow \mathrm{W}$. any $\mathrm{b}, \mathrm{r}=0$, or $\varphi(r)<\varphi(\mathrm{b})$.

Definition $2.7 \quad \varphi: \mathrm{R} \rightarrow \mathrm{W}$, any $\mathrm{b}, \mathrm{r}=\mathrm{b}$, or $\varphi(r)<\varphi(\mathrm{b})$.

Here. we note that if $R$ has an identity then by taking to be the identity homomorphism our definitions are the same as the definitions from Amano [2]. Lonstra [7]. Kanemitsu and Yoshida [6], Nagata [10] [11], Sanuel [12

There are five other possible definitions with the maps $\varphi: R \backslash\{0\} \rightarrow W$ and $\varphi: R \rightarrow W$, but we can not define an algorithm for any of these.

Definition 2.8 $\varphi: \mathrm{R} \backslash\{0\} \rightarrow \mathrm{W}$, any $\mathrm{b}, \mathrm{r}=0$, or $\varphi(r)<\varphi(\mathrm{b})$.

Definition $29 \varphi: \mathrm{R} \backslash\{0\} \rightarrow \mathrm{W}$, any $\mathrm{b}, \mathrm{r}=\mathrm{b}$, or $\varphi(r)<\varphi(\mathrm{b})$. Definition $210 \hat{q}: R \backslash\{0\} \rightarrow W$. any b. $\varphi(r)<\varphi(b)$.

Definition $2.11 \varphi: R \backslash\{0\} \rightarrow W, b \neq 0, \varphi(r)<\varphi(b)$.

Definition $2.12 \quad \varphi: \mathrm{R} \rightarrow \mathrm{W}$, any b, $\varphi(r)<\varphi(\mathrm{b})$.

Here the first three definitions may be eliminated by taking $\mathrm{a} \neq 0$ and $\mathrm{b}=$ 0 since $\varphi(0)$ is not defined. The last two cases are not given algorithms by (hoosing $b$ where $\varphi(\mathrm{b})$ is minimal in $\mathrm{W}$.

Let us nean by the word " equivalent " that if $\varphi$ is an Euclidean algorithm according to one definition then it will be a Euclidean algorithm in the sense of the other. Then the connections between these seven new definitions are the same with the results of our paper on Euclidean rings i1). Here we only need to consider the elements of $\mathrm{R}$ with $\theta$ according to the definitions. By allowing $W$ and $\varphi$ to change all these definitions become 'culuivalent. Then we have the following picture 


$$
1 \Leftrightarrow 2 \Leftrightarrow 3 \Leftrightarrow 4 \Leftrightarrow 5 \Leftrightarrow 6 \Leftrightarrow 7
$$

where $i \in\{1,2, \ldots .7\}$ is Definition $2 . i$. Hence we need to use only one of these definitions. say Definition 2.6 to define a Euclidean ring.

Definition 213 Let $\mathrm{R}$ be a commutative ring. If there exists a commutative ring with identity $\mathrm{R}^{\prime}$, a $\mathrm{G}$-mononorphism $\theta: \mathrm{R} \rightarrow \mathrm{R}^{\prime}$, a well-ordered set $\mathrm{W}$. an algorithm $\varphi: \mathrm{R} \rightarrow \mathrm{W}$ satisfying one of Definitions 2.1-2.7, say Definition 2.6, then we say that $\mathrm{R}$ is a Euclidean ring with respect to $\theta: \mathrm{R} \rightarrow \mathrm{R}^{\prime}$ and $\varphi: \mathrm{R} \rightarrow \mathrm{W}$.

We consider now the special case where the well-ordered set is taken to be $Z^{+} \cup\{0\}$. In general Definition 2.5 does not imply Definition 2.6 and Definition 2.3 does not imply Definition 2.6 since the well-ordered set cannot (hange. However Definition 2.3 does imply Definition 2.5 since $\mathrm{Z}^{+} \cup\{0\}$ does not lave maximal element. Therefore we have the following picture in the caste of $\mathrm{Z}^{+} \cup\{0\}$.

$$
\begin{gathered}
1 \Leftrightarrow 2 \Leftrightarrow 3 \Leftrightarrow 4 \Leftrightarrow 5 \\
\Uparrow \\
6 \Leftrightarrow 7
\end{gathered}
$$

Therefore, the case of $Z^{+} \cup\{0\}$ splits up in the two cases corresponding to Definitions 2.1-2.5 ankl Definitions 2.6-2.7.

Hence overall we have three types of Euclidean ring as.

(i) Definition (iroup) A: Euclidean rings with respect to a general wellordered set using Definitions 2.1-2.7.

(ii) Definition Group B: Euclidean rings with respect to $Z^{+} \cup\{0\}$ using Definitions 2.1-2.5.

(iii)Definition Group C: Euclidean rings with respect to $\mathrm{Z}^{+} \cup\{0\}$ using Defintitions 2.6-2.7.

Here the Group) ('Euclitean rings come naturally into the picture, and these are equivalent to bounded Euclidean'rings (defined by Fletcher when the ring lias an identity [3]).

Now we give sone examples:

(i) Consider Z. $\varphi: Z \backslash\{0\} \rightarrow Z^{+} \cup\{0\}$ is an algorithm (with respect to $1: Z$ $\rightarrow \mathrm{Z})$ given by $\varphi(\mathrm{r})=|\mathrm{r}|$ according to Group B. But $\varphi: Z \backslash\{0\} \rightarrow \mathrm{Z}^{+} \cup\{0\}$ is not an algorithm (with respect to $1: \mathrm{Z} \rightarrow \mathrm{Z}$ ) according to Group $\mathrm{C}$ since we have a contradiction when $\mathrm{a} \neq 0$ and $\mathrm{b}=0$ since $\varphi(\mathrm{r})=\varphi(\mathrm{a})>\varphi(\mathrm{b})$. 
(ii i Conmede nZ with ine: $n Z \rightarrow Z$ and $\varphi^{-}: n Z \backslash\{0\} \rightarrow Z^{+} \cup\{0\}$ given by

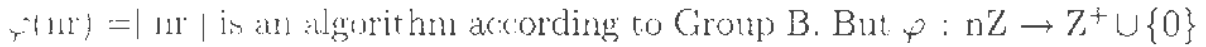
with the same matpping is not an algorithne according to Group $C$. The proof uf this is inmodiate by following the caso of $Z$ in ( $i$ ).

i ii ) Wo knw that $n \mathrm{Z} \times \mathrm{Z}$ is commutatiwe ring with identity with respect w t b following oprations for $\left(a_{1}, b_{1}\right),\left(a_{2}, b_{2}\right) \in n Z \times Z$,

$$
\begin{aligned}
\left(a_{1}, b_{1}\right)+\left(a_{2}, b_{2}\right) & =\left(a_{2}+b_{1}, a_{2}+b_{2}\right) \text { and }\left(a_{1}, b_{1}\right)\left(a_{2}, b_{2}\right) \\
& =\left(a_{1} a_{2}+b_{2} a_{1}+b_{1} a_{2}+b_{1} a_{2}\right)
\end{aligned}
$$

Nom. consider $\mathrm{nZ}$ with a $\mathrm{G}$-monomorphisn $\theta: \mathrm{nZ} \rightarrow \mathrm{nZ} \times \mathrm{Z}$ given by $\theta(n)$. (nI, 0$)$ and $p: n Z \backslash\{0\} \rightarrow Z^{-} \cup\{0\}$ is an algorithn (with respect $1 w 0: n Z-n Z \times Z)$ given $b y(n)-n \mid$ according to Group B. For all

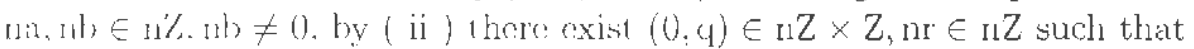

$$
\theta(\mathrm{na})=(0, \mathrm{~g}) \theta(\mathrm{nb})-\theta(\mathrm{nr}) \text { where } n \mathrm{n}=0 \text { or } \varphi(\mathrm{nr})<\varphi(\mathrm{nb}) .
$$

But $\varphi^{2}: n Z \rightarrow Z^{\top} \cup\{0\}$ given by $\varphi(n r)=|n r|$ is not an algorithm (with rempert $10 \theta: n Z, n Z \times Z$ ) according to Group $C$ since we cannot have III $\mid={ }_{r}^{r}\{n r\}<r(0)=0$ for $n b=0 . n r=n a \neq 0$.

(ir) Consider the subring $R=\{0,2,4,6\} \subset Z_{8}$. $R$ is a Euclidean ring in the sense of Group $C$ with respeset to inc: $: \mathrm{R} \rightarrow \mathrm{Z}_{8}$ and algorithm $q: R \rightarrow Z^{\prime} L\{(0\}$ given by $\varphi(r)=r(r \neq 0\}, \varphi(0)=7$.

If $R$ is a communation ring with iclentily them $R$ is a Euclidean ring if and osly if it is a Euclideas ling (with respect to $1: R \rightarrow R$ ). Therefore

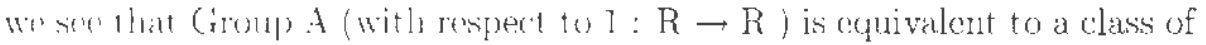
the definitions from Antano. Lenstra. Niggat a. Sammel. Group B and Group C (with respest to $1: R \rightarrow R$ ) are equivalent to Fletcher's definition and his bounded Euclidean ring respectively .

We have smen that there are fundanentally three types of Eurlidean ring R. These threg gromps are distinct. An example of a Euclidean ring Group

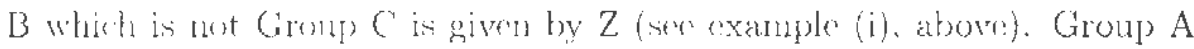

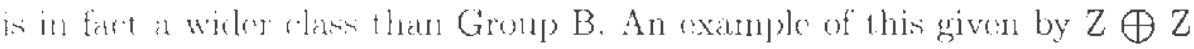
that is a Eudidedrn ring Group A but not Eudidean ring Group B (see [11] ankl 3 .) 


\section{The generalization of Motzkin's work}

We generalize Wotzkin work to conmutative rings (not necessarily with identity $\left[53\right.$. Suppose $\mathrm{R}$ is any commut ative ring, $\mathrm{R}^{\prime}$ a commut ative ring

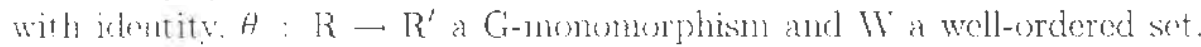

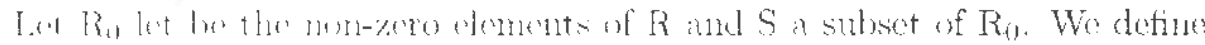

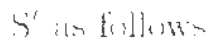

$$
\left.s^{\prime}-\{s \in s:]_{\mathrm{t}} \in \mathrm{R} \text { such that } \theta(\mathrm{a})-\theta(s) \mathrm{R}^{\prime} \leq \theta(s)\right\} \text {. }
$$

Thxu $S^{\prime} S$ and if $S_{1} \subseteq S$ we have $S_{1}^{\prime} \subseteq S^{\prime}$ sinco $x \in S_{1}^{\prime}$ implian $\theta(a)+$ $\theta(x) R^{\prime} \subseteq \theta(S:) \subseteq \theta(S)$ and $x \in S^{\prime}$. Here, also wo noto that a $\subseteq S$ necossarily sins $11 i^{-} R^{\prime}$.

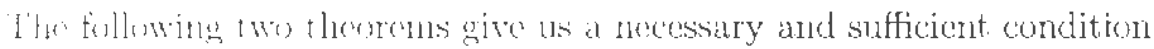
for a enmantive ring to bo Euclidean in the semse of the three groups.

Theorem 31 Suppose $R$ is a Fuclictean ring Group $A$ (Definition 2.6) with rospest to $\theta: R \rightarrow \mathrm{R}^{\prime}$ and the algorithm $\varphi: \mathrm{R} \rightarrow \mathrm{W}$. Let $\mathrm{P}_{\lambda}$ be the set of $\mathrm{x} \in$ Ro such that $\varphi(\mathrm{x}) \geq \lambda\left(\lambda \in \mathrm{W}_{,} 0 \leq \lambda \leq \mu\right.$ where $\varphi\left(0_{\mathrm{H}}\right)=\mu$ and 0 denotes the least elenent of $W$. Then tho subsets $\left\{P_{\lambda}\right\}$ have the following prumetics: (i) $P_{\lambda} \subset P_{\lambda-1}$. but $P_{\lambda} \subseteq \bigcap_{a<\lambda} P_{\alpha}$ if $\lambda-1$ does not exist, (ii)

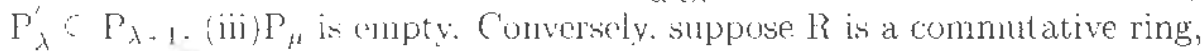
f) $^{\prime} \mathrm{R} \rightarrow \mathrm{R}^{\prime} \mathrm{n}$ G-mononomphism and $\mathrm{W}$ a well-ordered set. If there are subsets $\left\{I_{\lambda}^{\prime}\right\}$ of $R_{0}(\lambda \in W$ ) satisfyirg (i)-(iii) ther $R$ is Euclidean Group $\mathrm{A}$ (with respect to $\theta: \mathrm{R} \rightarrow \mathrm{R}^{\prime}$ and there exists an algorithm $\varphi: \mathrm{R} \rightarrow \mathrm{W}$ givm by $P(x)=\lambda$ if and only if $x \in P_{\lambda} \backslash P_{\lambda+1}$.

Proof (i) Cherly if $\lambda-1 \in W$ then $P_{\lambda} S_{\lambda-1}$ for all $\lambda \in W$ such that $0 \leq \lambda \leq \mu=\psi(0)$. If $\lambda-1$ does not uxist then for $x \in P_{\lambda}, \varphi(x) \geq \lambda$. Thim inplies $x(x) \geq \beta$ for $\alpha<\lambda$. Therefore $x \in P_{\alpha}$, for $\alpha<\lambda$ and hence

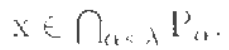

(ii Simpuose $b \in \mathrm{P}_{\lambda}^{\prime}$ and $a \in R$ such that $\theta(a)+\theta(b) R^{\prime} \subseteq \theta\left(\mathrm{P}_{\lambda}\right)$. Since $R$ is a Fulditcan ring. there exist. $\mathrm{q}^{\prime} \in \mathrm{R}^{\prime}$ andr $\in \mathrm{R}$ such that

$$
\theta(a)=q^{\prime} \theta(b)+\theta(1) \text { where } r=0 \text { or } \varphi(r)<\varphi(b) .
$$

If $\mathrm{r}=0$ then $\| \in \theta(\mathrm{a})+\theta(\mathrm{b}) \mathrm{H}^{\prime} \subseteq \theta\left(\mathrm{P}_{\lambda}\right)$. This is a contradiction. Therefore $\varphi(v)<\varphi(b)$. Sircc $\theta(a)+\theta(b) R^{\prime} \subseteq \theta\left(\mathrm{P}_{\lambda}\right), \quad \theta(\mathrm{r}) \in \theta\left(\mathrm{l}_{\lambda}\right)$ and this implies $\varphi(\mathrm{r}) \geq \lambda$. Therefore $\varphi(\mathrm{b}) \geq \lambda+1$ and hence $\mathrm{b} \in \mathrm{P}^{2} \lambda+1$.

(iii) Finally suppose $x \in P_{\mu}$, then $\varphi(x) \geq \mu$. Since $R$ is a Euclidean ring for $x, 0 \in R$ there oxist $q^{\prime} \in R^{\prime}$ and $r \in R$ such that

$$
H(x)=y^{\prime}\left(0+\theta(r) \text { where } r^{2}(r)<p(0) \text { and } r=x \neq 0\right. \text {. }
$$


Therefore $\psi(x)<\psi(0)=\mu$ which is a contradiction. Hence $\mathrm{P}_{\mu}$ is empty.

Comversely. take $a, b \in R$. If $b \mid a$ then

$$
\theta(\mathrm{a})=\theta(\mathrm{b}) \theta(\mathrm{q})+\theta(\mathrm{r}) \text { where } \mathrm{r}=0 \text { and } \mathrm{q} \in \mathrm{R} .
$$

So. suppose b does not divide a and choose $\mathrm{q}^{\prime} \in \mathrm{R}^{\prime}$ such that $\varphi(\mathrm{a}+\mathrm{c})$ is minimal and $\theta(c)=\theta(b) q^{\prime}$. Let $\varphi(a+c)=a$. Thus if $\theta(\mathrm{d})=\theta(\mathrm{b}) \mathrm{s}$ for some $s \in \mathrm{R}^{\prime}$ then $\varphi(\mathrm{a}+\mathrm{d}) \geq \alpha$ and $\theta(\mathrm{a})+\theta(\mathrm{b}) \mathrm{R}^{\prime} \subseteq \theta\left(\mathrm{P}_{\alpha}\right)$. If $\varphi(\mathrm{b})=\gamma$ we must prove $a<\gamma$. Suppose not, then $\alpha \geq \gamma$ and $\mathrm{P}_{\alpha} \subseteq \mathrm{P}_{\gamma}$. But $\mathrm{b} \in \mathrm{P}_{\gamma}$ and $\theta(\mathrm{a})+\theta(\mathrm{b}) \mathrm{R}^{\prime} \subseteq \theta\left(\mathrm{P}_{\alpha}\right) \subseteq \theta\left(\mathrm{P}_{\gamma}\right)$. which implies $\mathrm{b} \in \mathrm{P}_{\gamma}^{\prime} \subseteq \mathrm{P}_{\gamma+1}$. This is a contradiction. i.e. $a<$ ) .

Remark In the (ase of $\mathrm{W}^{\prime}=\mathrm{Z}^{+} \cup\{0\}$ we have Theoren 3.1 with the same prosf fur (immp (. Here. (i) becomes $P_{i} \subseteq P_{-1}$ for $i \in Z^{+}$since $i-1$ always 'xists.

Theorem 32 Suppose $\varphi: R \backslash\{0\} \rightarrow Z^{+} \cup\{0\}$ defines Euclidean algorithm in the sense of Group $B$ ( with respect to $\theta: R \rightarrow R^{\prime}$ ). Let $P_{i}$ be the set of $x \in R_{0}$ where $\varphi(x) \geq i(i=0,1, \ldots)$. Then subsets $\left\{P_{i}\right\}$ have the following properties:

(i) $R_{0}=P_{0} \supseteq P_{1} \supseteq P_{2} \supseteq \ldots$.. (ii) $P_{i}^{\prime} \subseteq P_{i+1}$. (iii) $\bigcap_{i=0}^{\infty} P_{i}$ is empty. Conversely, suppese $R$ is a commutative ring and $\theta: R \rightarrow R^{\prime}$ a G-monomorphism. If there are subsets $\left\{P_{i}\right\}$ of $R_{0}\left(i \in Z^{+} \cup\{0\}\right.$ ) which satisfying (i)-(iii) then $\mathrm{R}$ is Euclidean Group B (with respect to $\theta: \mathrm{R} \rightarrow \mathrm{R}^{\prime}$ ) and there exists an algorithm $\varphi: R \backslash\{0\} \rightarrow \mathrm{Z}^{+} \cup\{0\}$ given by $\varphi(\mathrm{x})=\mathrm{i}$ if and only if $\mathrm{x} \in \mathrm{P}_{\mathrm{i}} \backslash \mathrm{P}_{\mathrm{i}+1}$.

Proof Suppose first we have a Euclidean algorithm in the sense of Group B. ('learly $P_{i, 1} \subseteq P_{i}$ for all $\mathrm{i} \geq 0$. Now suppose $\mathrm{x} \in \bigcap_{\mathrm{i}=0}^{\infty} P_{\mathrm{i}}$ and let $\varphi(\mathrm{x})=\mathrm{n}$ then $x \notin P_{n+1}$. This is a contradiction. Hence $\bigcap_{i=0}^{\infty} P_{i}$ is empty. Finally, suppose $\mathrm{b} \in \mathrm{P}_{\mathrm{i}}^{\prime}$ and let $\mathrm{a} \in \mathrm{R}$ be such that $\theta(\mathrm{a})+\theta(\mathrm{b}) \mathrm{R}^{\prime} \subseteq \theta\left(\mathrm{P}_{\mathrm{i}}\right)$. Now there exist $\mathrm{q}^{\prime} \in \mathrm{R}^{\prime}$ and $\mathrm{r} \in \mathrm{R}$ with property that

$$
\theta(\mathrm{a})=\mathrm{q}^{\prime} \theta(\mathrm{b})+\theta(\mathrm{r}) \text { where } \mathrm{r}=0 \text { or } \varphi(\mathrm{r})<\varphi(\mathrm{b}) .
$$

If $r=0$ th' w w have $0=\theta(\mathrm{a})-\theta(\mathrm{b}) \mathrm{q}^{\prime} \in \theta(\mathrm{a})+\theta(\mathrm{b}) \mathrm{R}^{\prime} \subseteq \theta\left(\mathrm{P}_{\mathrm{i}}\right)$ and hence $\| \in \mathrm{P}_{\mathrm{i}}$. this is a contradiction. Therefore $\varphi(r)<\varphi(b)$. Since $\theta(\mathrm{a})+\theta(\mathrm{b}) \mathrm{R}^{\prime} \subseteq$ $\theta\left(\mathrm{P}_{\mathrm{i}}\right), \theta(\mathrm{r}) \in \theta\left(\mathrm{P}_{\mathrm{i}}\right)$ and this implies $\varphi(\mathrm{r}) \geq \mathrm{i}$. Therefore $\varphi(\mathrm{b})>\mathrm{i}$ and $b \in P_{i+1}$.

For the converse we have a similar proof as in the previous proof.

The subsets $\left\{\mathrm{P}_{\lambda}\right\}$, like the mapping $\varphi: R \rightarrow W$ can be defined in a number of ways. To make this idea more useful we define one set of 


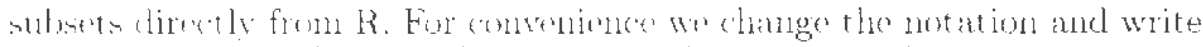

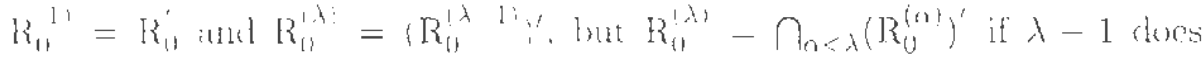

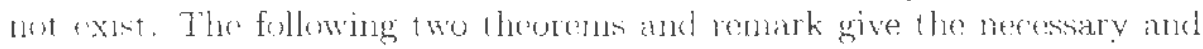
sutficicont conditions for the there grenpar.

Theorem $33 R$ is a Euclideatu ring Group $A$ ( with repext to $\theta: R \rightarrow R^{\prime}$ )

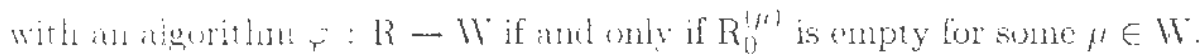
Fiurther if $R$ is Euclidom Gromp $A$ (with respect to $0: R \rightarrow R^{\prime}$ and if $\left\{P_{\lambda}\right\}$

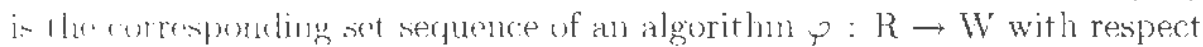
to $\theta: R \rightarrow R^{\prime}$ (destined in Theoren 3.1). then $R_{0}^{(\lambda)} \subseteq P_{\lambda}$ for all $\lambda \in W$ and

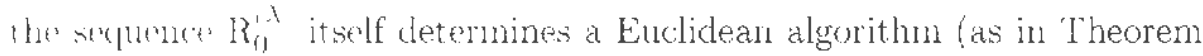
3.1 .

Proof We hise The rem 3.1. Let us suppose $R_{0}^{\{1\rangle}$ is empty for some $\mu \in W$. Then (iii) is watisliexl. Since $\mathrm{S}^{\prime} \subseteq \mathrm{S}$ for any subset $\mathrm{S}$, if $\lambda-1 \in \mathrm{W}$ by the definition $\mathrm{R}_{0}^{(\lambda)}=\left(\mathrm{R}_{0}^{(\lambda-1)}\right)^{\prime} \subseteq \mathrm{R}_{0}^{(\lambda-1)}$. If $\lambda-1$ does not exist, then by the definition $R_{0}^{(\lambda)}=\left(\bigcap_{c<<\lambda} R_{0}^{(\alpha)}\right)^{\prime} \subseteq \bigcap_{\alpha<\lambda \lambda} R_{0}^{(\alpha)}$ and hence (i) satisfied. Also, In the definition we have $\left(R_{(1}^{(\lambda)}\right)^{\prime}=R_{0}^{(\lambda-1)}$ and therefore (ii) satisfied with arplatity:

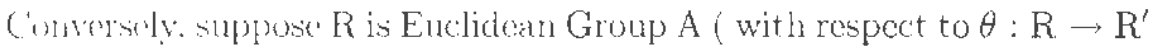
and $y$ which gives subsets $\mathrm{P}_{\alpha}$ ). Ther $\mathrm{R}_{0}-\mathrm{P}_{0}, \mathrm{R}_{0}^{(1)}=\mathrm{R}_{0}^{\prime}=\mathrm{P}_{0}^{\prime} \subseteq \mathrm{P}_{1}$ and hy induction $R_{0}^{(\lambda)} \subseteq P_{\lambda}$ for all $W$. Therefore $R_{0}^{(\mu)} \subseteq P_{\mu}$ and by Theorem 3.1 property (iii) $R_{0}^{(\mu)}$ is enpty. Hence from Theorem 3.1 the subsets $\left\{R_{0}^{(\lambda)}\right\}$ antoruine as Euclud an algorithn in the sense of Group A (with respect to $\left.\theta: R \cdot \mathrm{R}^{\prime}\right)$

Remark By taking $W=Z^{+} \cup\{0\}$, wo have the equivalence of Theorem 3.3 For Grom $\mathrm{C}$.

Theorem 3.4 R is a Euclidean ring Group B (with respcet to $\theta: \mathrm{R} \rightarrow \mathrm{R}^{\prime}$ ) ) if and only if $\left(\cap_{-0)}^{\infty} R_{0}^{(i)}\right)$ is enpty. Firther if $R$ is Enclidean Group $B$ (with respect to $\theta: R \rightarrow R^{\prime}$ and if $\left\{P_{i}\right\}$ are the corresponding sets of an algurithun of $\mathrm{R}$ with respect $100: \mathrm{R} \rightarrow \mathrm{R}^{\prime}$ (defined in Theoren 3.2) then $k_{0 i}^{(i)} \subset \mathrm{P}_{\mathrm{i}}$ for all $\mathrm{i} \geq 0$ and. the sequence $\left\{\mathrm{R}_{0}^{(i)}\right\}$ it self deternines a Euclidean allyorithun (as in Theorm 3.2).

Proof Use Theorm 3.2 and the same way as in the previons proof.

\section{Definition 3.5}

(i) Two ordered set.s $\mathrm{S}$. T are said to be order-isomorphic if there exists a bijuction $f: S \rightarrow T$ which presserver the ordering, i.c. $a \leq b \Leftrightarrow f(a) \leq f(b)$. 
(ii) let $\varphi_{1}: R \rightarrow H_{1}$ and $\varphi_{2}: \mathrm{R} \rightarrow \mathrm{W}_{2}$ be two algorithms on a ring $\mathrm{R}$ (with respret to $\theta: R \rightarrow R^{\prime}$ ). If there exists an order-isomorphism map $\mathrm{f}: \varphi_{1}(R) \rightarrow \varphi_{2}(R)$ such that $\varphi_{2}=$ fo $\varphi_{1}$ then we say that $\varphi_{1}$ and $\varphi_{2}$ are isomorphic algorithms.

Since every well-ordered set with cardinal $\leq$ card $(R)$ is order-isomorphic to an initial segment of any well-ordered set with cardinal $>$ card $(\mathrm{R})$ all algorithms on a ring $R$ maty be construed to take their values in a fixed well-ordered set. Let $W_{R}$ be a well-ordered set of ordinals whose cardinality deese not exeend the cardinality of $\mathrm{R}$.

Definition 3.6 Suppose R is Euclidean ring Groups A, C (or Group B). Then the algorithnn defined by the $\mathrm{R}_{0}^{(\mathrm{i})}$ which was given in Theorem 3.3 (or in Therem 3.4) is (alled the smallest algoritm in the sense of Groups A, ('or Group B).

Vote that we mulst define $p(0)$ for Definitions 2.3-2.7. For Definitions 2.3 and 2.4 we cand define $\varphi(0)$ as the least element of W. For Definitions 2.6-2.7 we define $\varphi(0)=\mu$ as in Theorem 3.3. In fact this is the unique maximal clement of $\varphi(R)$. The only problem is in the sense of Definition 2.5. In this (atse. to get the algorithm with the smallest values we increase each $\varphi(\mathrm{r})$ by 1 and put $\varphi(0)=0$ whiclu is the least element of W. Some examples follow shortly:

The following theorem shows us the connection between the smallest algorithms with respect to Definitions 2.3, 2.6 and 2.5.

Theorem 37 Suppose $R$ is a Euclidean ring Group A (with respect to $\left.\theta: \mathrm{R} \rightarrow \mathrm{R}^{\prime}\right)$.

(i) If $r_{3}: R \rightarrow W_{R}$ and $\varphi_{6}: R \rightarrow W_{R}$ are the smallest algorithms wrt. Detinitions 2.3 and 2.6 respectively, then $\varphi_{3}(\mathrm{r})=\varphi_{6}(\mathrm{r})$ for all non-zero $r \in R$.

(ii)If $\varphi_{: 3}: 13 \rightarrow W_{\mathrm{R}}$ and $\varphi_{5}: \mathrm{R} \rightarrow \mathrm{W}_{\mathrm{R}}$ are the smallest algorithms wrt. Definitions 2.3 and 2.5 respectively, then for all $\mathrm{r} \in \mathrm{R}, \varphi_{3}(\mathrm{r}) \leq \varphi_{5}(\mathrm{r}) \leq$ $4: 3(1)+1$ when right hand side exists.

Therefore we "an define the smallest algorithm either in terms of Definition 2.3 (\%) Definition 2.6 .

Proof (i) It is clear that every algorithm with respect to Definition 2.6 is an algorithn witlı respect to Definition 2.3. Therefore $\varphi_{6}$ is an algorithm with respec to Definition 2.3 and hence $\varphi_{3}(\mathrm{r}) \leq \varphi_{6}(\mathrm{r})$ for all $\mathrm{r} \in \mathrm{R}(1)$. Define $\zeta_{3}: R \rightarrow W_{R}$ by $\zeta_{3}(r)=\varphi_{3}(r)(r \neq 0), \zeta_{3}(0)=\varphi_{6}(0)$.

Then $\zeta_{3}$ is an algorithm with respect to Definition 2.6. For suppose $\mathrm{a}, \mathrm{b} \in$ $R$. If $l, \neq 0$ then there exist $q^{\prime} \in R^{\prime}$ and $\in R$ such that 
$H(\mathrm{c})=\theta(b) a_{1}^{\prime}+\theta(\mathrm{r})$ where $\mathrm{r}=0$ or $\zeta_{3}(\mathrm{r})=\varphi_{3}(\mathrm{r})<\varphi_{3}(\mathrm{~b})=\zeta_{3}(\mathrm{~b})$.

If $b=0$ then eavily we have

$\theta(\mathrm{a})=0+\theta(\mathrm{r})$ where $\mathrm{r}=\mathrm{a}=0$ or $\zeta_{3}(\mathrm{r})=\varphi_{3}(\mathrm{r}) \leq \varphi_{6}(\mathrm{r})<\varphi_{6}(0)=\zeta_{3}(0)$.

Since $\varphi_{i}$ is the smallest algorithm with respect to Definition 2.6 , we have for all num-zero $r \in R \varphi^{2}(\mathrm{r}) \leq \zeta_{3}(\mathrm{r})=\varphi_{3}(\mathrm{r})(2)$

Therefore. he $(1)$ and $(2) p_{3}: 3(r)=\varphi_{6}(\mathrm{r})$ for all non-zero $\mathrm{r} \in \mathrm{R}$.

(ii) It in straightforward to (heck that $\varphi_{5}$ is an algorithm with respect to Definition 2.3. Therefore $\varphi_{3}(\mathrm{r}) \leq \varphi_{5}(\mathrm{r})$ for all $\mathrm{r} \in \mathrm{R}$. Suppose $\varphi_{3}(\mathrm{r})+1$ exists in $W_{R}$ for all $r \in R$. Define $\zeta_{3}: R \rightarrow W_{R}$ by $\zeta(r)=\varphi_{3}(r)+1(r \neq$ $0), \zeta_{3}(0)=$ min. of $W_{\mathrm{R}}$. Then $\zeta_{3}$ is an algorithm with respect to Definition 2.5. For suppose $a, b \in R$. If $b \neq 0$, there exist $q^{\prime} \in R^{\prime}$ and $r \in R$ such that $\theta(\mathrm{a})=\theta(\mathrm{b}) \mathrm{q}^{\prime}$ where $\mathrm{r}=0$ or $\varphi_{3}(\mathrm{r})<\varphi_{3}(\mathrm{~b})$.

Therefore by the definition of $\zeta_{3}$ we have $\theta(\mathrm{a})=\theta(\mathrm{b}) \mathrm{q}^{\prime}+\theta(\mathrm{r})$ where $\zeta_{3}(\mathrm{r})<$ 4 (b). Since $y_{5}$ is the snallest algorithm with respect to Definition 2.5, we have $\gamma_{5}(\mathrm{r}) \leq \zeta_{3}(\mathrm{r})$ for all $\mathrm{r} \in \mathrm{R}$. In fact wo write $\zeta_{3}(\mathrm{r}) \leq \varphi_{3}(\mathrm{r})+1$ for all $\mathrm{r}$ $\in R$. Therefore $\varphi_{5}(\mathrm{r}) \leq \varphi_{3}(\mathrm{r})+1$ and hence $\varphi_{3}(\mathrm{r}) \leq \varphi_{5}(\mathrm{r}) \leq \varphi_{33}(\mathrm{r})+1$ for all $\mathrm{r} \in \mathrm{R}$.

We show that the smallest algorithm satisfies an inequality which is given by the following theorem. Here, we give Theorem 3.8 with respect to (iroup) A. but also the same theorem with the same proof is given wrt. (iroups $B$ and $C$ by taking $W=Z^{+} \cup\{0\}$.

Theorem 38 If $\mathrm{R}$ is a Euclidean ring Group $\mathrm{A}$ (with respect to $\theta: \mathrm{R} \rightarrow \mathrm{R}^{\prime}$ ) then the smallest algorithm $\varphi: R \rightarrow W$ defined by the subsets $\left\{R_{0}^{(\lambda)}\right\}$ satisfies the following property $\varphi(b) \geq \varphi(a)$ for all $a, b \in R$ where $b \neq 0$ and $\theta(b)=\theta(a) q^{\prime}$ for $q^{\prime} \in R^{\prime}$.

Proof Suppose $\varphi(\mathrm{b})=\lambda$ and $\varphi(\mathrm{a})=\alpha$. Therefore $\mathrm{b} \in \mathrm{R}_{0}^{(\lambda)} \backslash \mathrm{R}_{0}^{(\lambda+1)}$ and $\mathrm{a} \in$ $\mathrm{R}_{0}^{(\alpha)} \backslash \mathrm{R}_{0}^{(\alpha+1)}$. If $\varphi(\mathrm{b})<\varphi(\mathrm{a})$ then $\lambda<\alpha$ and $\mathrm{R}_{0}^{(\lambda)} \supset \mathrm{R}_{0}^{(\alpha)}$ with strict inclusion since $\bigcap_{\lambda=0}^{\infty} R_{0}^{(\lambda)}$ is empty (because $R_{0}^{(\mu)}$ is empty for some $\mu \epsilon$ W). Suppose $\alpha-1$ exists in W. Since $a \in R_{0}^{(\alpha)}$ there exists $y_{1} \in R$ such that $\theta\left(\mathrm{y}_{1}\right)+\theta(\mathrm{a}) \mathrm{R}^{\prime} \subseteq \theta\left(\mathrm{R}_{0}^{(\alpha-1)}\right) \subseteq \theta\left(\mathrm{R}_{0}^{(\lambda)}\right)$. Suppose now, $\alpha-1$ does nut exist. Since a $\in R_{0}^{(\alpha)}=\left(\bigcap_{\gamma<\alpha} R_{0}^{(\gamma)}\right)^{\prime}$ there exists $y_{2} \in R$ such that $\theta\left(\mathrm{y}_{2}\right)+\theta(\mathrm{a}) \mathrm{R}^{\prime} \subseteq \theta\left(\bigcap_{\gamma<\alpha} \mathrm{R}_{0}^{(\gamma)}\right) \subseteq \theta\left(\mathrm{R}_{0}^{(\lambda)}\right)$. Therefore, there exists $\mathrm{y} \in \mathrm{R}$ wuch that $\theta(\mathrm{y})+\theta(\mathrm{b}) \mathrm{R}^{\prime}=\theta(\mathrm{y})+\theta(\mathrm{a}) \mathrm{q}^{\prime} \mathrm{R}^{\prime} \subseteq \theta\left(\mathrm{R}_{0}^{(\lambda)}\right)$ and $\mathrm{b} \in \mathrm{R}_{0}^{(\lambda+1)}$. This is a contradiction. Hence $\varphi(\mathrm{a}) \geq \varphi(\mathrm{a})$.

Corollary If $\mathrm{R}$ is a Euclidean ring Group $\mathrm{A}$ (with respect to $\theta: \mathrm{R} \rightarrow \mathrm{R}^{\prime}$ ) then the smallest algorithm $\varphi: R \rightarrow W$ defined by the subsets $\left\{R_{0}^{(\lambda)}\right\}$ satisfies the following property 
(ab) $\geq$ (a) for all a. b $\mathrm{C} R$ where $a b \neq 0$.

Theorem 3.8 therefore gonoralises the incquality property given by Fletcher [3.

Suppose $R$ is a romentutative ring with identity. Consider the G-monomor-

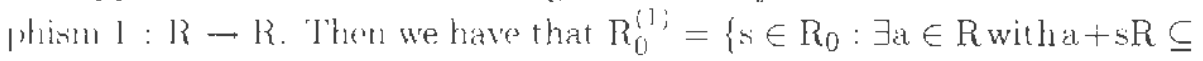

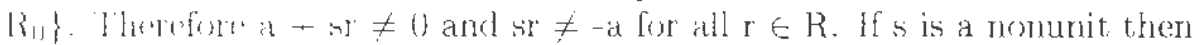
watake $a:-1$ arded $s \in R_{0}^{(1)}$. If $s$ is a mut then for $r=-s^{-1}$ a we have $a+$ $\therefore I^{\prime}=\|$ and $\therefore \notin R_{0}^{(1)}$. Honce $R_{0}^{(1)}=R_{0} \backslash\{$ units $\}$.

Hore we gives some examples.

Example 1 (i) First. we ruay take the ring $\mathrm{R}=\mathrm{Z}_{12}$, show it is a Euclidean Jing Group $B$ (and Group $C$ ) with respest $101: Z_{12} \rightarrow Z_{12}$ and determine its stmallem ingurithn $\rightarrow$. Thereforo $\mathbb{R}_{0}=\{1,2,3, \ldots, 10,11\}$.

Lis whend above, $\mathrm{K}_{0}^{(1)}=\{2,3,4,6,8,9,10\}$.

ll is casy to show that $\mathrm{R}_{0}^{(2)}=\{4,6,8\}$. Because $2+4 \mathrm{R}, 2+6 \mathrm{R}, 2+8 \mathrm{R}$ $\subseteq \mathrm{I}_{0}^{(1)}$ and $\mathrm{a}-\mathrm{sR} \subseteq \mathrm{R}_{0}^{(1)}$ for all $\mathrm{a} \subseteq \mathrm{R}_{0}^{(1)}$ and $s \in\{2,3,9,10\}$. Also $\mathrm{R}_{0}^{(3)}$ is ampty since $x+4 R \subseteq R_{0}^{(2)}, y+6 R \subseteq R_{0}^{(2)}$ and $z+8 R \subseteq R_{0}^{(2)}$ for all $x y, z \in R_{0}^{(2)}$. Thus $Z_{12}$ is a Euclidean ring in the sense of Group $B$ (and

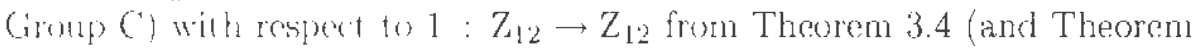
3.31 .

(ii) Vur. lu un consider the subring $\mathrm{R}=\{0.2,4.6,8.10\} \subset \mathrm{Z}_{12}$ with the inclusion thomonomplism ine. $\mathrm{R} \rightarrow \mathrm{Z}_{12}$. By the definition $R_{0}^{11}=\left\{s \subseteq R_{0}: \exists a \subseteq R\right.$ with $\left.\theta(a)+\theta(s) R^{\prime} \subseteq \theta\left(R_{0}\right)\right\}$ and $R_{0}=\{2,4,6,8,10\}$. Cloarlythero is no a $\in R$ with $a+2 Z_{12} \subseteq R_{0}$. Therefore $2 \notin R_{0}^{(1)}$. For $2 \in Z_{12}$ wo kaw $2+1 Z_{12}=\{2.6 .10\} \subset R_{0}$ and $4 \in R_{0}^{(1)}$. Similarly, $2+6 Z_{12}=$ $\{2 . s\} \subset R_{11}, 2-s Z_{2}=\{2,6,10\} \subset R_{0}$ and hence $6,8 \in R_{0}^{(1)}$. There is no

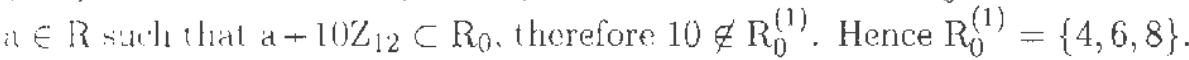
Since there is no $x, y, z \in R$ such that $x+1 Z_{12}=x+\{2,6,10\} \subset R_{0}^{(1)}, y+$ $6 Z_{12}=y+\{0,6\} \subset R_{0}^{(1)}$ and $z+8 Z_{12}=z+\{0,4,8\} \subset R_{0}^{(1)}, 4,6,8 \notin R_{0}^{(2)}$. Therefore $R_{0}^{(2)}$ is enpty. Honce $R$ is uclidean ring Group $B$ (and Groupe) witl, rosuret to inc : $R \rightarrow Z_{12}$ and its smallet algorithm $\varphi$ is given by

$$
p(2)=0, p(4)=1, \varphi(6)=1, \varphi(8)=1, \varphi(10)=0
$$

(I) Fur an algorithru in the sense of Definitions 2.3-2.4, wo take $\varphi(0)=0$.

$$
\varphi(1)=0 . \varphi(2)=0 . \varphi(4)=1, \varphi(6)=1, \varphi(8)=1, \varphi(10)=0 .
$$

(I1) For and algorithn in the serse of Definitions 2.6-2.7, we take $\varphi(0)=2$,

$$
\varphi(11)=2 . \varphi(2) \cdots 0 . \psi(4)=1 . \varphi(6)=1, \varphi(8)=1, \psi(10)=0 .
$$


Alw in the wene of Defintition 2.5 to get the algorithm with smallest values

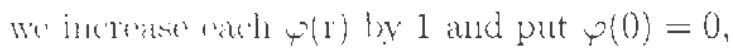

$$
\varphi(0)=0 . \varphi(2)=1 . \varphi(4)=2, \varphi(6)=2, \varphi(8)=2, \varphi(10)=1 \text {. }
$$

Example 2 For a positive integer 11 , consider the ring $n \%$ with the $G$ nunumorphismo

(i) inc. : $\mathrm{nZ} \rightarrow \mathrm{Z}$ and (ii) $\theta: \mathrm{nZ} \rightarrow \mathrm{nZ} \times \mathrm{Z}$.

The smallest algorithuls (with respect to these G-monomorphisms) are given $b y-(n r)=k$ for $11 \mathrm{r} \neq 0$ where $12^{k} \leq|n r|<n 2^{k+1}$ (i.e $2^{k} \leq 1 \mathrm{r} \mid<2^{\mathrm{k}+1}$ 1. For wo prowe by induction will Motzkin s notation that

$$
(117,(1))^{(k)} \quad\left\{+112^{k}, \pm n\left(2^{k}+1\right), \pm n\left(2^{k}+2\right), \ldots\right\} .
$$

Note that $11 Z_{11}=\{ \pm 11,+211, \pm 3 n, \ldots\}$. For $k=1$ let us prove $\left(n Z_{0}\right)^{(1)}=$ $\{=211, \pm 311 \ldots\}$. Bexause if there exists na $\in \mathrm{nZ} Z_{0}$ snch that na $+( \pm n) Z \subseteq$ $n Z_{0}$

(ancl for (ii) (nd. 0$\left.)-( \pm n, 0)(n Z \times Z) \subseteq \theta\left(n Z_{0}\right)\right)$ then $0 \in$ na $\pm n Z$ (and for (ii)

(11.11] $C(11 \mathrm{a} .0)) \pm(n, 6)(n \mathrm{Z} \times \mathrm{Z}))$ which is a contradiction. Therefore $\pm \mathrm{n} \notin$ $(n \%, 1)^{i l:}$. Fin

$|\mathrm{nII}|>11 . \mathrm{nI} \in\left(\mathrm{n} Z_{0}\right)^{(1)}$ since $\pm \mathrm{n} \pm \mathrm{nr}^{\prime} \mathrm{Z} \subseteq \mathrm{nZ}$ ( (and $\pm(\mathrm{n}, 0) \pm(\mathrm{nr}, 0)(\mathrm{nZ} \times \mathrm{Z}) \subseteq$ $\left.\theta\left(n Z_{0}\right)\right)$. Suppose now $\left(n Z_{0}\right)^{(k)}=\left\{ \pm n 2^{k}, \pm n\left(2^{k}+1\right), \pm n\left(2^{k}+2\right), \ldots\right\}$ and let us prove $\left(n Z_{0}\right)^{(k+1)}=\left\{ \pm n 2^{k+1}, \pm n\left(2^{k+1}+1\right), \pm n\left(2^{k+1}+2\right), \ldots\right\}$. Take $n h \in\left(n Z_{0}\right)^{(k)}$ with, $\mathrm{nb} \mid<\mathrm{n} 2^{\mathrm{k}+1}$ and suppose there exists na $\in \mathrm{nZ}$ such that $n m-n h t, \subseteq\left(n Z_{0}\right)\{k\}$

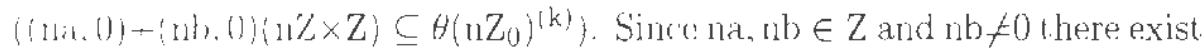

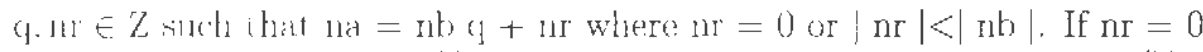
then $0 \in n a+n b Z \subseteq\left(n Z_{0}\right)^{(k)}\left((0,0) \in(n a, 0)+(n b, 0)(n Z \times Z) \subseteq \theta\left(\left(n Z_{0}\right)^{(k)}\right)\right.$, this is a contradiction. Suppose now $\mid$ nir $|<|$ nb $\mid$. IIere there are the two (atices:

(I) If $|n n|<n 2^{k}$ then we have a contradiction since $n$ In $\in$ na + nbZ $\subseteq$

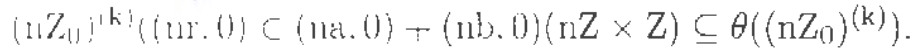

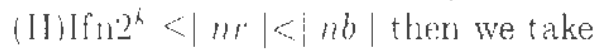

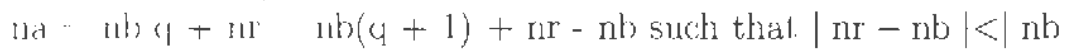
(if $|n r-n b| \geq \mid$ ulb $\mid$ then we consider as na $=n b(q-1)+n r+n b$ with $\mid$ nIr + nlb $\rangle|<| n b \mid$ and continue similarly $)$. In fact $|n r-n b|<n 2^{k+1}-n 2^{k}$ and therefore wo have

$n r-n b \in n a+n b Z \subseteq\left\langle n Z_{0}\right)^{(k)}((n r-n b, 0) \in(n a, 0)+(n b, 0)(n Z \times Z) \subseteq$ $\left(\left(11 \%_{11}\right)^{(k)}\right)$ 
which is a contradiction. Thus for $|\mathrm{nb}|<\mathrm{n} 2^{\mathrm{k}+1}, \mathrm{nb} \notin\left(\mathrm{nZ} \mathrm{Z}_{0}\right)^{(\mathrm{k}+1)}$. If $\mid n b) \mid \geq n 2^{k+1}$ wo easily take na $=n 2^{k} \in n Z$ and we have

$$
\mathrm{na}+\mathrm{nbZ} \subseteq\left(\mathrm{nZ} \mathrm{Z}_{0}\right)^{(\mathrm{k})}\left((\mathrm{na}, 0)+(\mathrm{nb}, 0)(\mathrm{nZ} \times \mathrm{Z}) \subseteq \theta\left(\left(\mathrm{nZ} \mathrm{Z}_{0}\right)^{(\mathrm{k})}\right) .\right.
$$

Hen' $\left(n Z_{(1)}\right)^{(k+1)}=\left\{ \pm n 2^{k+1}, \pm n\left(2^{k+1}+1\right), \pm n\left(2^{k+1}+2\right), \ldots\right\}$

Fur instance. $\varphi^{-}(14)=1 . \varphi(42)=2, \varphi(70)=3$ are some values of the smallest algorithm $\varphi: 7 \mathrm{Z} \rightarrow \mathrm{Z}^{+} \cup\{0\}$ (with respect to $\theta: 7 \mathrm{Z} \rightarrow 7 \mathrm{Z} \times$ Z). If 175 and 7() are given two elements in $7 Z$ then we have $\theta(175)=$ $\theta(70)(3,-1)+\theta(35)$ where $\varphi(35)=2<\varphi(70)$.

\section{References}

$1]$ Agargun, A.G., and Fletcher, C.R., "Euclidean rings", Doa Tr. J. Math.. 19. pp. $291-399,(1995)$.

[ 2 ] Amano, K., "A note on Euclidean ring", Bull. Fac. Gen. Ed. Gifu Univ.. No.20. pp. 13 - 15, (1985).

[ 3 j Fletcher. C.R., "Euclidean rings". J. London Math. Soc., (2), 4, 79-82, (1971).

4 ; Hiblot. J.J.. "Des anneaux euclidiens dont le plus petit algorithme II'st pas valeurs finies". Comptes Rendues, 281, srie A-411-414, (1975).

[ 5 ] Hiblot. J.J.. "Correction une note sur les anneaux euclidiens", Comptes Rendues.284, srie A-847, (1977).

16! Kancmitsu. M.. and Yoshida, K., "Euclidean rings", Bull. Fac. Sci., Iharaki Lnir.. Math.. No.18. (1986).

7) Lenstra. H.W.. Lect ures on Euclidean Rings, Bielefeld, Summer (1974).

[४] Motzkin, T., "The Euclidean algorithm", Bull. Amer. Math. Soc., 55, pp. $1142-1146,(1949)$.

[ 9 ] Nagata. M., “On Euclidean algorithm". Tata Inst. Fund. Res. Studies in Nath.. 8. p). 175 - 186. (1978).

101' Nagata. M.. "Some remarks on Euclid rings", J. Math. Kyoto Univ., pp. $25-3$. pp. $421-422$. (1985).

[11] Nagata, M., "On the definition of a Euclidean ring", Advanced Studies in Pure Mathematics. 11, pp. $167-171,(1987)$.

'12j Samuel. P.. "About Euclidean rings", Journal of Algebra, 19, pp. $282-$ 3(1) (1971). 
Roroind: Soptomber 20, 1995

\author{
Ahmet Goksel Agargun \\ Yildiz Trohnical Universitesi \\ Ftri-Ed. Fikultomi Matomatik Bolumu \\ Ogratim loumi \\ Sisli. Istambul \\ Turkey
}

